\title{
Associations between the duration of active commuting to school and academic achievement in rural Chilean adolescents
}

\author{
Antonio García-Hermoso ${ }^{1,2+}$, Jose M. Saavedra ${ }^{3}$, Jordi Olloquequi ${ }^{4}$ and Robinson Ramírez-Vélez ${ }^{5^{*}+}$
}

\begin{abstract}
Background: Habitual active commuting to school may be positively associated with academic achievement. The aim of this study was to examine the relationship between duration of walking or otherwise actively commuting to school and academic achievement.

Methods: This cross-sectional study included 389 adolescents from seven rural schools (12-13 years). Mode and duration of active commuting to school (use of active means such as walking or biking to and from school) and screen time were self-reported. Academic achievement was determined by the outcome in basic grades (language and mathematics).

Results: Active commuting to school was not associated with higher scores in any grades after adjustment for potential confounders. No evidence was found of interactions between gender and academic achievement, but there was interaction with duration of walking ( $<30 \mathrm{~min}, 30-60 \mathrm{~min}$, and $>60 \mathrm{~min}$ ). Adjusted binary logistic regression analysis suggested that adolescents who spent between 30 and 60 min actively commuting were more likely to obtain high academic achievement (language and mathematics).

Conclusions: Thirty to 60 min of ACS may have a positive influence on academic achievement in adolescents, so, it is necessary to make recommendations for the children to walk from and/or to school. This could help society to recognize the relevance of physical activity to health as well as to academic performance.
\end{abstract}

Keywords: Active travel, Physical activity, Walking, School performance

\section{Background}

Cultural shifts and changes in home and neighbourhood environments discourage physical activity (PA) in young people [1]. Active commuting to school (ACS), defined as the use of active means such as walking or biking to and from school, is an inexpensive form of PA that can be integrated into adolescents' routines [2]. It has been argued that if sufficient intensity is achieved, ACS could lead to an increase in cardiovascular fitness [3] and is associated with a healthier body composition and metabolic profile [4]. Several studies suggest that adolescents

\footnotetext{
* Correspondence: robinson.ramirez@urosario.edu.co; robin640@hotmail.com ${ }^{\dagger}$ Equal contributors

${ }^{5}$ Centro de Estudios para la Medición de la Actividad Física «CEMA». Escuela de Medicina y Ciencias de la Salud, Universidad del Rosario, Cra. 24 No. 63C 69, Bogotá, DC, Colombia

Full list of author information is available at the end of the article
}

who actively commute to and from school can accumulate an additional $20 \mathrm{~min}$ of moderate to vigorous PA across the whole day, compared to adolescents who use passive transport [5].

The evidence presented in a recent review suggests that health markers (including physical activity, nutrition, and body composition) affect the structure and function of the hippocampus, a structure known to be critical for successful learning and remembering [6]. Given that studies suggest that PA has positive effects on academic achievement [7] and cognitive performance in adolescents [8], habitual ACS may be positively associated with these as previously the evidence have suggested [9]. These works have studied the relationship between ACS and academic achievement in an adolescent population. For example, Martinez-Gomez et al. [9] reported a positive association between ACS 
and cognitive performance in urban Spanish adolescent girls, especially in those who reported more than 15 min of ACS per day compared to girls who spent fewer than 15 min. However, this study [9] evaluated cognitive performance with SRA Test of Educational Ability which measured in the general way verbal (command of language), numeric (speed and precision in performing operations with numbers and quantitative concepts), and reasoning (the ability to find logical ordination criteria in sets of numbers, figures, or letters) abilities.

Youth from rural areas and small cities were more active than urban children, although the differences were small to moderate [10]. Regarding ACS, evidence suggests that youth who live in rural areas are less likely to actively commute to school than those living in urban areas [11], which may be related to fewer pedestrian infrastructures, longer commuting routes, and poorer access to public transport. Despite the growing interest in active travel to and from school, none studies have explored the duration of walking ACS and its relationship with academic achievement from rural areas. To the best of our knowledge, the ACS-academic achievement relationship has not been assessed in a Latin American country at the rural population level. Thus, the aim of this study was to examine the relationship between duration of walking ACS and academic achievement in rural Chilean adolescents. We hypothesize that in rural areas the duration of walking ACS has a direct relationship with the academic achievement, i.e. big duration, big academic achievement.

\section{Methods}

\section{Study design and participants}

Data collection took place between March and June 2014. All students $[n=454]$ from seventh grade schools in the Maule region (Chile) were invited to participate. In seventh grade the students meet their thirteenth in the academic year. The schools were selected for accessibility (convenience sampling consisted of selecting schools that could most easily or willingly participate in this study). Subjects were excluded if they had special educational needs [learning difficulties and/or learning disabilities] or had any type of dysfunction limiting their PA (any disease or problem). Physical education teachers provided this information. Also, due to the small number of adolescents $(n=6)$ who commuted actively to or from school by cycling, they were excluded. Thus, we analyzed only subjects who commuted actively to or from school by walking. Finally, a sample of 389 adolescent students (12-13 years, $86 \%$ of invited), 196 boys and 193 girls, agreed to participate in the study. The study protocol was approved by the (blinded for purposes of review) Ethics Committee and complied with the principles of the Declaration of Helsinki. The study was conducted according to ethical standards in sport and exercise science research [12]. A letter was sent to parents of all adolescents in the seventh grade. It invited them to a meeting where the objectives were explained, after which they signed the informed consent for the participation of their adolescents in the study.

\section{Mode and duration of commuting to school}

The mode of commuting to and from school was measured by the self-reported questionnaire. Two questions were asked about the mode and duration of commuting to school: (1) "How do you usually travel from home to school and from school to home?" and (2) "How long does it usually take you to travel from home to school or from school to home?" Response options to the first question were walking, biking, bus, car/motorcycle, and others. Response options to the second question included $15 \mathrm{~min}$ or shorter, from 15 to $30 \mathrm{~min}$, from 30 to $60 \mathrm{~min}$, and longer than $60 \mathrm{~min}$. Responses were categorized as: ACS $\leq 30 \mathrm{~min}$, ACS 30-60 min, and ACS > $60 \mathrm{~min}$. If subjects reported at least one of the trips as active [i.e. to or from school], they were included in the active commuting group (ACS group). In contrast, if subjects not reported at least one of the trips as active, they were included in the non-active commuting group (non-ACS group).

Previous studies have demonstrated evidence of the reliability and validity for similar questions [13].

\section{Academic achievement}

Academic achievement was assessed using the students' grades in mathematics and language using a standard process within Chile public schools (same curriculum and contents). The grades were collected from the schools' official records at four time points in the first semester (March, April, May and June, 2014). In Chilean elementary schools, student grades range from 1 (worst) to 7 (best), classified as: very poor $=1.0-1.9$; poor $=2.0-2.9$; below average $=3.0-3.9$; average $=4.0$ 4.9 ; $\operatorname{good}=5.0-5.9$; very good $=6.0-7.0$. The average score was calculated, determining high academic achievement with a grade above or equal to 5.0 (good and very good scores) [14].

\section{Potential confounders}

At the beginning of each potential confounder, the reason for its inclusion in the study has been explained.

\section{Anthropometry}

Several studies have reported negative associations between obesity and school achievement [14]. For that reason, the body height and weight were measured with the adolescents having bare feet and wearing light 
underclothes, using an electronic scale (Seca, Berlin, Germany). Body mass index $\left(B M I=\mathrm{kg} / \mathrm{m}^{2}\right)$ and height for age were evaluated and $z$-scores were obtained according to the Centers for Disease Control and Prevention [15] references. Weight status was defined as follows: underweight $(z$-score $<-1 \mathrm{SD})$, normal weight ( $z$-score from $-1 \mathrm{SD}$ to $1 \mathrm{SD})$, at risk of obesity ( $z$-score from $>1 \mathrm{~s}$ to $<2 \mathrm{SD})$ and obese $(z$-score $\geq 2 \mathrm{SD})$.

\section{Screen time}

This parameter is inversely related to academic achievement [14]. So, three questions from the Health Behavior in School-Aged Children study were used [16] regarding daily television, videogame and computer use: 'About how many hours a day do you usually: (i) watch television; (ii) play computer or video games; (iii) use a computer [for purposes other than playing games-for example, emailing, chatting, or surfing the internet or doing homework] in your free time?' Daily screen time averages were calculated by adding the answers to these three questions together. Finally, screen time was dichotomized as recommended $(<2 \mathrm{~h} / \mathrm{d})$ and excessive $(\geq 2 \mathrm{~h} / \mathrm{d})$, based on the American Academy of Pediatrics' international guidance on limiting paediatric screen time [17].

\section{Physical activity}

A recent review suggests that PA is positively related to academic achievement [18]. For that, the PA was measured with the self-administered Spanish version of the Physical Activity Questionnaire for Adolescents (PAQ-A) [19]. The questionnaire was designed to assess adolescents' levels of moderate and vigorous PA. Adolescents were asked to quantify their PA levels during their spare time in the previous week. Nine items scored on a fivepoint Likert scale were averaged to derive an overall PA score ranging from one to five (higher scores indicating higher levels of PA). Finally, physical activity was dichotomized as high (four quartile) and low-medium (first to third quartile).

\section{Maternal education}

The majority of the literature on parents' education pertains to the direct and positive influence on achievement for children and youths [20]. Mothers completed a questionnaire about their highest level of education and were dichotomized as having an education level below the university level or an education at or above the university [post high school] level.

\section{Socioeconomic status}

There is evidence indicating that socioeconomic status (SES) is one of the most important determinants of childhood school achievement [21]. Therefore the SES was obtained using a scale based on Graffar's modified method [22], which considers items such as schooling, job held by the head of the household and characteristics of the house, taking into account three categories (high, medium, and low SES).

\section{Neonatal characteristics}

Birth weight is associated with cognitive impairments persisting into childhood [23]. Therefore, the birth weight $(\mathrm{kg})$ was reported by parents and dichotomized as low birth weight $(\leq 2.500 \mathrm{~g})$ and normal birth weight $(>2.500 \mathrm{~g})$ [24].

\section{Data analysis}

Descriptive statistics were performed to characterize the sample. The continuous variables were expressed as the mean and standard deviation and frequency distribution for categorical data. Statistical normality was tested using the Kolmogorov-Smirnov test. Due to their skewed distribution, the language and mathematics variables were natural log-transformed. To aid interpretation, data were back-transformed from the log scale for presentation in the results to examine the associations between mode of commuting to school (non-ACS or ACS) and academic achievement. The analysis of covariance was conducted after adjusting for gender, weight status, screen time, PA, maternal education, SES, school, and birth weight. The interaction between gender and ACS categories (i.e., duration of ACS) was performed by means of a two-way ANOVA. This interaction term was not significant (data not shown) and so there was no stratification by gender. ANCOVA models were estimated to test differences in academic by ACS categories adjusted for sex, weight status, screen time, PA, maternal education, SES, school, and birth weight. Pairwise posthoc comparisons were examined using the Bonferroni test. Finally, a model was considered using high academic achievement (score $\geq 5.0$ ) as the dependent variable and the primary explanatory variable was the duration of ACS category. The model was adjusted variables abovementioned. Variable selection for the logistic regression models was also guided by the bivariate analyses. The Hosmer-Lemeshow goodness-of-fit test was used to assess the fit of the multiple logistic models. Data analysis was performed using IBM SPSS Statistics (version 22; SPSS, Inc., Chicago, IL, USA). A $p$-value of $\leq 0.05$ denoted statistical significance.

\section{Results}

Table 1 shows the adolescents' characteristics. The sample SES was predominantly (68.6\%) medium. Around $23 \%$ of the students made at least one trip (to or from school) by active means. There were no differences in academic achievement (language and mathematics) between walking ACS categories (non-ACS and ACS) 
Table 1 Descriptive characteristics for the participants in this study $(n=389)$

\begin{tabular}{|c|c|}
\hline Girls, n (\%) & $193(49.6)$ \\
\hline Age, years & $12.0 \pm 0.6$ \\
\hline \multicolumn{2}{|l|}{ Weight status $^{a}$} \\
\hline Underweight, $n(\%)$ & $4(1.0)$ \\
\hline Normal, $n(\%)$ & $172(44.2)$ \\
\hline Overweight, $n$ (\%) & $115(29.6)$ \\
\hline Obesity, n (\%) & $98(25.2)$ \\
\hline \multicolumn{2}{|l|}{ Birth weight } \\
\hline Low birth weight ( $\leq 2500 \mathrm{~g}), n(\%)$ & $20(5.1)$ \\
\hline \multicolumn{2}{|l|}{ Self-reported physical activity } \\
\hline High $^{b}$ & $72(18.5)$ \\
\hline \multicolumn{2}{|l|}{ Sedentary behaviour } \\
\hline Exceeding recommended limits ${ }^{c}, n(\%)$ & $266(68.4)$ \\
\hline \multicolumn{2}{|l|}{ Mother's education } \\
\hline Level university, $n$ (\%) & $57(14.7)$ \\
\hline \multicolumn{2}{|l|}{ SES } \\
\hline Low, $n(\%)$ & $52(13.4)$ \\
\hline Medium, $n(\%)$ & $267(68.6)$ \\
\hline High, $n(\%)$ & $70(18.0)$ \\
\hline \multicolumn{2}{|l|}{ Academic achievement } \\
\hline Language $(1-7)$ & $5.0 \pm 0.9$ \\
\hline High academic achievement, $n(\%)$ & $217(57.7)$ \\
\hline Mathematics (1-7) & $5.3 \pm 0.6$ \\
\hline High academic achievement, $n$ (\%) & $238(61.3)$ \\
\hline \multicolumn{2}{|l|}{ Means of transport } \\
\hline Public transport, $n(\%)$ & $148(38.0)$ \\
\hline Car/Motorbike, $n$ (\%) & $152(39.1)$ \\
\hline \multicolumn{2}{|l|}{ Frequency } \\
\hline Active transport to school, $n(\%)$ & $89(22.9)$ \\
\hline Active transport from school, $n(\%)$ & $111(28.5)$ \\
\hline \multicolumn{2}{|l|}{ Duration of ACS ${ }^{d}$} \\
\hline Non-ACS, n (\%) & $273(70.2)$ \\
\hline ACS $\leq 30 \mathrm{~min}, n(\%)$ & $55(14.1)$ \\
\hline ACS 30-60 min, $n(\%)$ & $40(10.3)$ \\
\hline $\mathrm{ACS} \geq 60 \mathrm{~min}, n(\%)$ & $21(5.4)$ \\
\hline
\end{tabular}

Values are means (standard deviations $\pm \mathrm{SD}$ ) and number and proportions (\%) for categorical data. ACS, active commuting to school; BMI, body mass index; SES, socio economic status. ${ }^{a}$, Weight status defined as follows: Underweight (z-score $<-1 \mathrm{~s})$, Normal weight (z-score from $-1 \mathrm{~s}$ to $1 \mathrm{~s}$ ), Obesity risk (z-score from $1 \mathrm{~s}$ to $2 \mathrm{~s}$ ) and Obesity (z-score $>2 \mathrm{~s}$ ); ${ }^{\mathrm{b}}$, Physical activity $\geq 2.09{ }^{\mathrm{c}}{ }^{\mathrm{C}}, \geq 2 \mathrm{~h} /$ day of

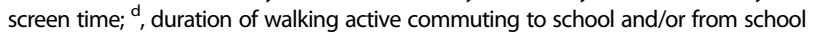

adjusted for sex, weight status, screen time, PA, maternal education, SES and birth weight: language $(5.02 \pm 0.43$, non-ACS vs $5.12 \pm 0.45$, ACS; $p=0.836$ ) and mathematics $(5.14 \pm 0.42$, non-ACS vs $5.58 \pm 0.65$, ACS; $p=0.677)$.

Figure 1 shows mean differences in academic achievement by categories of ACS. Academic attainment was

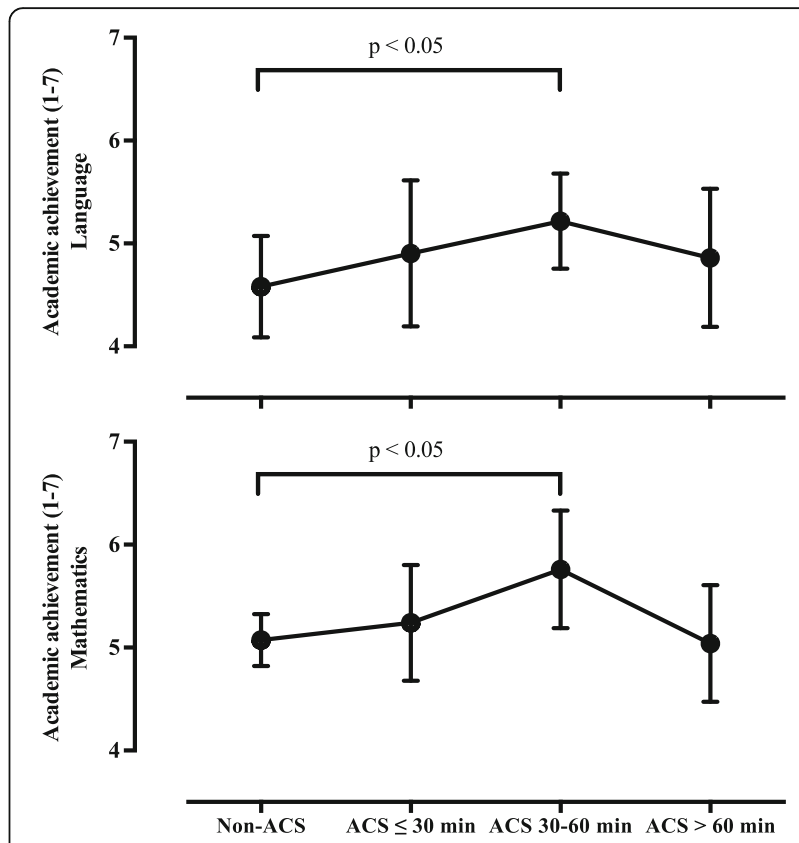

Fig. 1 Mean differences in academic achievement by categories of active commuting to school. ACS, active commuting to school

higher in adolescents with 30 to $60 \mathrm{~min}$ of ACS than non-commuters in language $(p=0.016)$ and mathematics $(p=0.031)$

Table 2 shows the OR of the relationship between academic achievement and duration of ACS. In both grades, adjusted analysis suggests that students with 30 to $60 \mathrm{~min}$ of ACS were more likely to have a better academic achievement than non-commuters (language, OR $=3.53,95 \%$ CI, 1.12 to $4.37 ; p=0.003$; mathematics, $\mathrm{OR}$ $=2.19,95 \% \mathrm{CI}, 1.06$ to $5.05 ; p=0.028)$. The HosmerLemeshow goodness-of-fit test suggests that each of the models fit reasonably well, with $p$ values 0.818 and 0.933 in language and mathematics, respectively.

Although differences by sex, weight status, and screen time are not the main focus of this article, some comments should be made on their effect on the outcome variables. In Chile, the male students traditionally show better performance in the standardized tests, but in this study they were more likely to perform better in language, but not in mathematics. On the other hand, obese adolescents had worse grades than their counterparts with lower adiposity in language. Finally, adolescents who exceeded two hours of screen time daily had worse mathematics grades than adolescents who meet the screen time recommendations.

\section{Discussion}

The aim of this cross-sectional study was to analyse the relationship between duration of walking ACS and academic achievement in rural Chilean adolescents. The 
Table 2 Logistic regression analyses for duration of walking ACS associated with academic achievement in Chilean adolescents, after adjusting for sex, weight status, birth weight, physical activity, screen time, maternal education, and SES

\begin{tabular}{|c|c|c|c|c|}
\hline & \multicolumn{2}{|l|}{ Language } & \multicolumn{2}{|l|}{ Mathematics } \\
\hline & OR $(95 \% \mathrm{Cl})$ & $p$ & OR $(95 \% \mathrm{Cl})$ & $p$ \\
\hline \multicolumn{5}{|l|}{ Sex } \\
\hline Girls & 1.00 & & 1.00 & \\
\hline Boys & 2.09 (1.19 to 3.67$)$ & 0.010 & 1.19 (0.71 to 2.01$)$ & 0.506 \\
\hline \multicolumn{5}{|l|}{ Weight status } \\
\hline Normoweight & 1.00 & & 1.00 & \\
\hline Overweight + obesity & 0.64 (0.40 to 0.95$)$ & 0.023 & 0.77 (0.46 to 1.31$)$ & 0.343 \\
\hline \multicolumn{5}{|l|}{ Birth weight } \\
\hline$>2500 \mathrm{~g}$ & 1.00 & & 1.00 & \\
\hline$\leq 2500 \mathrm{~g}$ & $0.22(0.04$ to 1.24$)$ & 0.085 & 0.88 (0.34 to 2.28$)$ & 0.869 \\
\hline \multicolumn{5}{|c|}{ Self-reported physical activity } \\
\hline Low-Medium & 1.00 & & 1.00 & \\
\hline High & $1.45(0.76$ to 2.77$)$ & 0.261 & 1.35 (0.71 to 2.56$)$ & 0.353 \\
\hline \multicolumn{5}{|c|}{ Screen time recommendation } \\
\hline$<2$ h/day & 1.00 & & 1.00 & \\
\hline$\geq 2$ h/day & 0.98 (0.59 to 1.65$)$ & 0.962 & 0.25 (0.13 to 0.50$)$ & $<0.001$ \\
\hline \multicolumn{5}{|l|}{ Mother's education } \\
\hline Below university level & 1.00 & & 1.00 & \\
\hline University level & 0.66 (0.30 to 1.43$)$ & 0.290 & 1.38 (0.67 to 2.83$)$ & 0.375 \\
\hline \multicolumn{5}{|l|}{ SES } \\
\hline Low, n (\%) & 1.00 & & 1.00 & \\
\hline Medium, $n(\%)$ & 0.67 (0.19 to 2.43$)$ & 0.547 & $1.80(0.45$ to 7.30$)$ & 0.406 \\
\hline High, $n(\%)$ & 1.38 (0.59 to 2.80$)$ & 0.433 & 1.08 (0.68 to 1.73$)$ & 0.737 \\
\hline \multicolumn{5}{|c|}{ Active commuting to school } \\
\hline Non-ACS & 1.00 & & 1.00 & \\
\hline $\mathrm{ACS} \leq 30 \mathrm{~min}$ & $1.32(0.72$ to 2.41$)$ & 0.363 & 1.20 (0.69 to 2.07$)$ & 0.523 \\
\hline ACS $30-60 \mathrm{~min}$ & 3.53 (1.12 to 3.37$)$ & 0.003 & 2.19 (1.06 to 5.05$)$ & 0.028 \\
\hline ACS $\geq 60 \mathrm{~min}$ & 0.37 (0.06 to 2.21$)$ & 0.277 & 0.54 (0.12 to 2.48$)$ & 0.433 \\
\hline Likelihood ratio $\left(\mathrm{Chi}^{2}\right)$ & 116.18 & $<0.001$ & 40.27 & $<0.001$ \\
\hline Hosmer-Lemeshow & 3.66 & 0.818 & 2.423 & 0.933 \\
\hline Correctly classified (\%) & 72.2 & & 63.7 & \\
\hline Observations & 389 & & 389 & \\
\hline
\end{tabular}

results suggest that adolescents who spent 30 to $60 \mathrm{~min}$ on ACS were more likely to achieve a high academic achievement compared to non-ACS, independent of potential confounders (sex, weight status, PA, screen time, maternal education, SES, school, and birth weight).

The ACS represented $23 \%$ of the total time spent moving per week, and in addition to the health benefits of PA, neuroscience evidence also suggests that PA may exert a positive effect on children's cognitive functioning [functional brain changes], brain physiology (changes in regional brain volume are associated with increased peripheral brain-derived neurotrophic factor and memory) and academic achievement [25]. Also, a recent crosssectional study does support a positive relationship between PA and mathematics at 16 years [7]. Studies have reported that adolescence is a sensitive period to stimulate cognitive function and improve learning and academic achievement; however, in Chile it is the period of life with the greatest decline in PA levels [26]. Therefore, ACS is an opportunity to increase daily PA levels and consequently cognitive performance at school. The results support that ACS, adjusted by potential confounders, is not associated with academic achievement in either boys or girls. A recent study observed that 
when combined with obesity, low-medium levels of PA and excessive screen time might be related to poor academic achievement [14]. In this sense, obesity is associated with poor executive functioning, which is critical for academic achievement [27]. On the other hand, evidence has confirmed our results and have not observed a relationship between ACS and academic achievement [28].

Questions regarding the appropriate dose of ACS required to produce optimal outcomes in cognitive performance and academic achievement remain unanswered. A long distance to school implies a decreased likelihood of adopting active transport practices [29] and a distance of approximately two kilometres is associated with the best PA outcomes related to active transport [30]. Regarding duration, Martinez-Gómez et al. [9] reported that adolescent girls who spent more than 15 min on ACS had better cognitive performance variables than those who spent less time ( $\leq 15 \mathrm{~min})$ and non-commuters. The results suggested that adolescents who spent 30 to $60 \mathrm{~min}$ on ACS were more likely to obtain high academic achievement compared to non-ACS adolescents, independent of potential confounders including PA and weight status. This relationship would have an inverted U-shape, where both too little $(\leq 30 \mathrm{~min})$ and too much ACS $(\geq 60 \mathrm{~min})$ has a non-positive association with academic achievement. This fact could be consider a novel finding, indicating range where the ACS has relationship with academic achievement. In this way, $60 \mathrm{~min}$ or more could indicate long trip to school. This oblige to children wake-up too much early and, probably to have an insufficient sleep that has influence in academic achievement [31]. On the other hand, $30 \mathrm{~min}$ or less could be consider a short time to achieve improvement. Possibly, if this time was of "brisk" walking (nearer of "physical fitness" concept) could be have positive effects [32].

Generally, studies support that only vigorous physical activity might have a crucial role in improving cognitive function and memory [33]. However, intensity associated with this routine is usually light or moderate, and it was suggested that this type of intensity plays a role in cognitive performance $[9,28]$. Therefore, the results combine with others stating that a longer duration of ACS may positively influence school achievement. The results also showed that adolescents who spent $60 \mathrm{~min}$ or more actively commuting to school were no more likely to obtain a high mean academic achievement. These results could be due to adolescents who walk for longer normally waking before others, which could influence school performance. In this vein, a recent study of Portuguese adolescents suggests that waking up early is a predictor of study methods, reading skills, motivation for study and overall performance [34]. In the same way, these students would arrive home more tired than others, and it could hinder their homework. Therefore, more prospective and experimental studies are needed to further elucidate the complex relationships between ACS and cognitive performance.

The study has several limitations. First, the study is a cross-sectional design, which does not allow for the drawing of conclusions on the causal direction of the associations. Second, the sample is not representative of the population, so it difficult to generalize the results across the Chilean adolescent population. However, the sample is relevant: it gives interesting information. Third, the ACS (mode and duration of commuting to school) and PA variables were self-reported and we have not assessed the distance to and from school; therefore, findings must be interpreted with caution. Fourth, only $23 \%$ of the students made at least one trip (to or from school) by active means, which limits the statistical power. Fifth, using school-based grades given by teachers is subject to bias. Finally, the intensity of the ACS was not evaluated and higher intensity level of ACS could be associated with higher grades [33].

\section{Conclusions}

The results of this study suggest that rural adolescents who spent 30 to $60 \mathrm{~min}$ on ACS were more likely to achieve a high academic achievement (language and mathematics) compared to non-ACS adolescents. Therefore, efforts to maintain and increase walking to school may be particularly relevant as this is likely to have a positive impact on adolescents' school achievement. However, future researches in this field are necessary, especially intervention studies to increase PA and academic achievement.

\section{Abbreviations}

ACS: Active commuting to school; PA: Physical activity; SES: Socioeconomic status

\section{Acknowledgments}

The authors would like to thank the schools, adolescent, and families for their participation and interest in the study.

\section{Funding}

Not applicable.

\section{Availability of data and materials}

"The study data have legal restrictions and ethical imposed by the authors' IRB (Autonomous University of Chile). The explanation of these restrictions is because they are children and adolescent students of Public Schools Chile. The initial contact point for collaborations is Professor Antonio GarcíaHermoso (antonio.garcia.h@usach.cl).

\section{Authors' contributions}

AG-H provided concept/idea/research design. All authors provided writing. $R R-V$, JO and JM provided data collection. RR-V provided data analysis, project management, and participants. JO \& JMS provided consultation (including review of manuscript before submission). All authors read and approved the final manuscript.

\section{Competing interests}

The authors declare that they have no competing interests. 


\section{Consent for publication}

I have obtained consent to publish from the participants (or legal parents or guardians for adolescent) to report individual patient data.

\section{Ethics approval and consent to participate}

The protocol was in accordance with the latest revision of the Declaration of Helsinki and current Chilean laws governing clinical research on human subjects. The Review Committee for Research on Human Subjects at the Autonomous University of Chile approved all of the study procedures. Informed consent and assent were obtained from all participants.

\section{Publisher's Note}

Springer Nature remains neutral with regard to jurisdictional claims in published maps and institutional affiliations.

\section{Author details}

${ }^{1}$ Laboratorio de Ciencias de la Actividad Física, el Deporte y la Salud, Facultad de Ciencias Médicas, Universidad de Santiago de Chile, USACH, Santiago de Chile, Chile. ${ }^{2}$ Facultad de Ciencias de la Educación, Universidad San Sebastián, Santiago de Chile, Chile. ${ }^{3}$ Physical Activity, Physical Education, Sport and Health Research Centre, Sports Science Department, School of Science and Engineering, Reykjavik University, Reykjavik, Iceland. ${ }^{4}$ Instituto de Ciencias Biomédicas, Facultad de Ciencias de la Salud, Universidad Autónoma de Chile, Talca, Chile. ${ }^{5}$ Centro de Estudios para la Medición de la Actividad Física «CEMA». Escuela de Medicina y Ciencias de la Salud, Universidad del Rosario, Cra. 24 No. 63C - 69, Bogotá, DC, Colombia.

\section{Received: 24 January 2017 Accepted: 7 March 2017}

\section{Published online: 04 April 2017}

\section{References}

1. Lobstein T, Baur L, Uauy R. Obesity in children and young people: a crisis in public health. Obes Rev. 2004;5(s1):4-85.

2. Cooper AR, Andersen LB, Wedderkopp N, Page AS, Froberg K. Physical activity levels of children who walk, cycle, or are driven to school. Am J Prev Med. 2005;29(3):179-84.

3. Shephard RJ. Is active commuting the answer to population health? Sports Med. 2008;38(9):751-8.

4. Pizarro AN, Ribeiro JC, Marques EA, Mota J, Santos MP. Is walking to school associated with improved metabolic health. Int J Behav Nutr Phys Act. 2013;10(1):12.

5. Southward EF, Page AS, Wheeler BW, Cooper AR. Contribution of the school journey to daily physical activity in children aged 11-12 years. Am J Prev Med. 2012:43(2):201-4

6. Hassevoort KM, Khan NA, Hillman CH, Cohen NJ. Childhood Markers of Health Behavior Relate to Hippocampal Health, Memory, and Academic Performance. Mind, Brain, and Education 2016. doi:10.1111/mbe.12108.

7. Booth J, Leary S, Joinson C, et al. Associations between objectively measured physical activity and academic attainment in adolescents from a UK cohort. Br J Sports Med. 2013:48(3):265-70.

8. Syväoja HJ, Tammelin TH, Ahonen T, Kankaanpää A, Kantomaa MT. The associations of objectively measured physical activity and sedentary time with cognitive functions in school-aged children. PLoS One. 2014;9(7): e103559.

9. Martínez-Gómez D, Ruiz JR, Gómez-Martínez S, et al. Active commuting to school and cognitive performance in adolescents: the AVENA study. Arch Pediatr Adolesc Med. 2011;165(4):300-5.

10. Joens-Matre RR, Welk GJ, Calabro MA, Russell DW, Nicklay E, Hensley LD. Rural-urban differences in physical activity, physical fitness, and overweight prevalence of children. J Rural Health. 2008;24(1):49-54.

11. Sjolie AN, Thuen F. School journeys and leisure activities in rural and urban adolescents in Norway. Health Promot Int. 2002;17(1):21-30.

12. Harriss D, Atkinson G. Update-ethical standards in sport and exercise science research. Int J Sports Med. 2011;32(11):819-21.

13. Evenson KR, Neelon B, Ball SC, Vaughn A, Ward DS. Validity and reliability of a school travel survey. J Phys Act Health. 2008:5(1):S1-S15.

14. García-Hermoso A, Marina R. Relationship of weight status, physical activity and screen time with academic achievement in adolescents. Obes Res Clin Pract 2015. 2017;11(1):44-50.

15. Kuczmarski RJ, Ogden CL, Grummer-Strawn LM, et al. CDC growth charts: United States. Adv Data. 2000;314:1-27.
16. Currie C. Social determinants of health and well-being among young people: world health organization regional office for Europe Copenhagen. 2012

17. Barlow SE. Expert committee recommendations regarding the prevention, assessment, and treatment of child and adolescent overweight and obesity: summary report. Pediatrics. 2007;120(Suppl 4):S164-92.

18. Fedewa AL, Ahn S, Erwin H, Davis MC. A randomized controlled design investigating the effects of classroom-based physical activity on children's fluid intelligence and achievement. Sch Psychol Int. 2015;36(2):135-53.

19. Martínez-Gómez D, Martínez-de-Haro V, Pozo T, et al. Fiabilidad y validez del cuestionario de actividad física PAQ-A en adolescentes españoles. Rev Esp Salud Pública. 2009:83(3):427-39.

20. Davis-Kean PE. The influence of parent education and family income on child achievement: the indirect role of parental expectations and the home environment. J Fam Psychol. 2005;19(2):294.

21. Lawlor DA, Najman JM, Batty GD, O'Callaghan MJ, Williams GM, Bor W. Early life predictors of childhood intelligence: findings from the Mater-University study of pregnancy and its outcomes. Paediatr Perinat Epidemiol. 2006; 20(2):148-62.

22. Álvarez ML, Muzzo S, Ivanovic D. Escala para medición del nivel socioeconómico, en el área de la salud. Rev Med Chile. 1985;113(3):243-9.

23. Esteban-Cornejo I, Tejero-González C, Castro-Piñero J, et al. Independent and combined influence of neonatal and current body composition on academic performance in youth: The UP \& DOWN Study. Pediatric Obes. 2015:10(3):157-64

24. Johnson EO, Breslau N. Increased risk of learning disabilities in low birth weight boys at age 11 years. Biol Psychiatry. 2000:47(6):490-500.

25. Voelcker-Rehage C, Niemann C. Structural and functional brain changes related to different types of physical activity across the life span. Neurosci Biobehav Rev. 2013;37(9):2268-95.

26. Ministry of Health. Global school-based survey. Department of Epidemiology. Santiago de Chile: Ministry of Health; 2007.

27. Miller AL, Lee HJ, Lumeng JC. Obesity-associated biomarkers and executive function in children. Pediatric Res. 2014;77(1-2):143-7.

28. Van Dijk ML, De Groot RH, Van Acker F, Savelberg HH, Kirschner PA. Active commuting to school, cognitive performance, and academic achievement: an observational study in Dutch adolescents using accelerometers. BMC Public Health. 2014:14(1):799.

29. Gutiérrez-Zornoza M, Sánchez-López M, García-Hermoso A, González-García A, Chillón P, Martínez-Vizcaíno V. Active Commuting to School, Weight Status, and Cardiometabolic Risk in Children From Rural Areas The Cuenca Study. Health Educ Behav. 2014: doi:10.1177/1090198114549373.

30. Duncan S, White K, Mavoa S, Stewart T, Hinckson E, Schofield G. Active transport, physical activity, and distance between home and school in children and adolescents. J Phys Act Health. 2016:13(4):447-53.

31. Li S, Arguelles L, Jiang F, et al. Sleep, school performance, and a schoolbased intervention among school-aged children: a sleep series study in China. PLoS One. 2013;8(7):e67928.

32. Donnelly JE, Hillman $\mathrm{CH}$, Castelli D, et al. Physical activity, fitness, cognitive function, and academic achievement in children: a systematic review. Med Sci Sports Exerc. 2016:48(6):1197-222.

33. Coe DP, Pivarnik JM, Womack CJ, Reeves MJ, Malina RM. Effect of physical education and activity levels on academic achievement in children. Med Sci Sports Exerc. 2006;38(8):1515

34. Duarte J, Nelas P, Chaves C, Ferreira M, Coutinho E, Cunha M. Sleep-wake patterns and their influence on school performance in Portuguese adolescents. Aten Primaria. 2014;46:160-4.

\section{Submit your next manuscript to BioMed Central and we will help you at every step:}

- We accept pre-submission inquiries

- Our selector tool helps you to find the most relevant journal

- We provide round the clock customer support

- Convenient online submission

- Thorough peer review

- Inclusion in PubMed and all major indexing services

- Maximum visibility for your research

Submit your manuscript at www.biomedcentral.com/submit
Biomed Central 